\title{
Watchful Waiting Strategy May Reduce Low-Value Diagnostic Testing
}

\author{
Larissa May, MD, MSPH, MSHS, Peter Franks, MD, MPH, Anthony Jerant, MD, \\ and Joshua Fenton, MD, MPH
}

Background: PCPs need effective communication strategies to address patient requests for low-value testing while sustaining patient-provider partnerships. Watchful waiting - allowing a negotiated period of time to pass before making a firm testing decision - shows promise as a tool for addressing patient requests for low-value testing.

Methods: Observational analysis of data from a randomized controlled trial of a communication intervention designed to boost patient-centeredness and reduce low-value test ordering among 61 resident primary care physicians. Intervention effectiveness was assessed during follow-up encounters of unannounced standardized patients (SPs) who requested low-value tests. We examined associations between five physician counseling behaviors and overall patient-centeredness (Measure of Patient-Centered Communication) and requested test ordering.

Results: During 155 SP encounters, residents most commonly used reassurance (96\% of encounters), evidence-based recommendations (97\%), and watchful waiting (68\%). Resident advice to pursue watchful waiting was associated with $39 \%$ lower likelihood of test ordering (adjusted marginal effect of $-38.6 \%$ [95\% CI -43.6 to -33.6$]$ ). When all communication behaviors were examined together, only watchful waiting was significantly associated with test ordering (marginal effect of $-38 \%$ [95\% CI $-44.3 \%$ to $-31.7 \%]$ ). Overall patient-centeredness was not associated with lowvalue testing.

Conclusion: Resident physician counseling to pursue watchful waiting was associated with less ordering of requested low-value diagnostic tests, while overall patient-centeredness was not. (J Am Board Fam Med 2016;29:710-717.)

Keywords: Communication; Counseling; Diagnostic Tests, Routine; Follow-up Studies; Physical Examination; Physicians, Primary Care; Probability; Risk; Watchful Waiting

Primary care physicians (PCPs) frequently order low-value diagnostic tests, ${ }^{1,2}$ which are by definition either inappropriate (they do more harm than good), unnecessary (they do neither good nor harm), or have low potential benefit but high

This article was externally peer reviewed.

Submitted 2 February 2016; revised 2 May 2016; accepted 9 May 2016.

From the Departments of Emergency Medicine (LM) and Family and Community Medicine (PF, AJ, JF), University of California-Davis, Sacramento, CA.

Funding: This study was funded by the Patient-Centered Outcomes Research Institute (grant "Promoting Patient Centered Counseling to Reduce Inappropriate Diagnostic Tests").

Conflict of interest: none declared.

Corresponding author: Larissa May, MD, MSPH, MSHS, Department of Emergency Medicine, University of California-Davis, 4150 v street, \# 2100, Sacramento, CA 95817 (E-mail: larissa.may@gmail.com). cost. PCPs recognize that low-value testing is problematic and widespread, even in their own practices. These and parallel findings in nonprimary care specialties have focused national attention on reducing low-value testing. ${ }^{3}$

While some low-value testing may be triggered by provider discomfort or diagnostic uncertainty, ${ }^{4,5}$ patients may also request low-value testing. Patient requests are ubiquitous in primary care, occurring during most visits, ${ }^{6}$ and the majority are fulfilled. ${ }^{7}$ Patients request diagnostic tests during approximately $10 \%$ of primary care visits, ${ }^{6}$ and many of these requested tests are likely to have a low value. PCPs perceive visits in which patients request testing as being more difficult, ${ }^{8}$ likely for several reasons. Some patients are anxious and want reassurance, ${ }^{9}$ and providers often rationalize low-value 
testing as a means of providing that reassurance, even though such testing does little to quell patient anxiety or reassure. ${ }^{10}$ Further, patients whose test requests are denied are less satisfied with their visits, ${ }^{7,11}$ a matter of increasing concern to PCPs, who often receive incentives based on patient experience scores. There is a need to uncover strategies PCPs can use to address patient requests for low-value testing while maintaining patient and provider satisfaction.

One such approach is to enhance the overall patient-centeredness of PCP communication. In patient-centered interactions, physicians explore patients' concerns and requests within a psychosocial context, with the goal of finding common ground from which evidence-based recommendations can be discussed and negotiated. ${ }^{12}$ Patient-centered counseling has been associated with reduced diagnostic testing without compromising patient satisfaction, ${ }^{1,13-16}$ suggesting that a more patient-centered approach may enable PCPs to meet the emotional and informational needs of patients who request lowvalue tests without acceding to testing.

However, the full patient-centered communication paradigm is multifaceted, complex, and associated with longer visits, ${ }^{14}$ and therefore is not always feasible to apply in toto. Among discrete patient-centered communication behaviors, recommending watchful waiting - allowing a negotiated period of time to pass before making a firm testing decision-shows promise as a tool for addressing patient requests for low-value testing. Most studies of watchful waiting have concerned treatment decisions, ${ }^{17,18}$ but 1 randomized controlled trial (RCT) examined its impact on low-value blood testing for unexplained medical symptoms. ${ }^{19}$ In the trial, conducted in the Netherlands, PCPs trained to recommend watchful waiting ordered fewer blood tests than controls, with no adverse impact on patient satisfaction or anxiety. It is unclear whether watchful waiting would be as effective and well received in the United States, which has higher per capita spending on diagnostic tests than the Netherlands and most other nations, or in other clinical scenarios.

To explore this issue, we conducted an observational analysis of data from an RCT that examined whether orders by resident PCPs for 3 low-value tests commonly ordered in the United Statesspinal magnetic resonance imaging (MRI) for sub- acute back pain, dual-energy $\mathrm{x}$-ray absorptiometry (DXA) screening for postmenopausal women at low risk for osteoporosis, and neuroimaging for recent-onset uncomplicated headache-could be reduced by training in patient-centered communication behaviors, including watchful waiting. ${ }^{20}$ The trial intervention did not affect the residents' use of watchful waiting, other targeted communication behaviors, or test ordering. However, given evidence that advice to pursue watchful waiting is associated with less testing in primary care settings in the Netherlands, we conducted an observational analysis examining the association of watchful waiting, other specific physician counseling behaviors (normalization, risks outweighing benefits, reassurance, and evidence-based recommendations for no testing), and patient-centered communication with low-value test ordering.

\section{Methods \\ Design and Setting}

This observational analysis used data from an RCT of an educational intervention delivered by standardized patient instructors (SPIs) during 2 simulated office visits with in primary care residents at the University of California, Davis Medical Center, Sacramento, CA. ${ }^{20}$ During these visits, SPIs spent about 20 minutes acting as a patient requesting low-value tests. SPIs then broke out of the patient role and provided personalized feedback to intervention physicians. Residents in the control group had visits with SPIs without personalized feedback. The clinical scenarios for the 2 SPI visits were a 48-year-old man with subacute back pain requesting a spinal MRI and a perimenopausal woman at low risk for osteoporotic fracture requesting DXA screening.

The overall goal of the intervention was to enhance the patient-centeredness of residents' responses to patient requests for low-value tests. In addition to enhancing overall patient-centeredness, the intervention emphasized 5 specific communication behaviors: (1) normalization; (2) reassuring patients by pointing to specific features of their history and physical examination; (3) explaining that the risks of testing outweigh the benefits in their situation; (4) suggesting watchful waiting such that testing will be ordered only if symptoms persist; and (5) advising evidence-based strategies to address patient concerns instead of requesting test- 
ing. Following the intervention, test ordering by physicians was measured during 3 unannounced standardized patient visits over the subsequent 3 to 12 months. Because we found no intervention effect on study outcomes or on specific communication behaviors, we conducted this post hoc observational analysis to assess associations between the 5 specific communication behaviors, overall patient-centeredness, and test ordering during the unannounced standardized patient visits. The study was approved by our institutional review board.

\section{Participants}

Participants were family medicine or internal medicine resident physicians who had completed $\geq 1$ year of residency and provided regular primary care at 1 of 2 primary care clinics at the university medical center. Residents were invited to participate in a study of "patient-doctor communication." Participants provided informed verbal consent and were randomly assigned to intervention and control groups.

\section{Standardized Patient Measurement Visits}

After SPI visits, residents saw up to 3 unannounced standardized patients (SPs) scheduled during regular clinic hours over a 3-to 12-month follow-up period, including visits with (1) a male patient with subacute back pain requesting spinal MRI; (2) a postmenopausal woman with fatigue requesting DXA screening; and (3) a 30-year-old woman with recent-onset headache requesting neuroimaging (to assess the generalization of intervention effects to other low-value tests). Using detailed case histories, 9 SPs were trained to convincingly portray patients, to request tests early during visits, and to accept omission of testing if residents persisted in declining their request. Using standardized forms, an SP supervisor prospectively assessed fidelity by listening to audio-recordings of selected visits, assessing role fidelity using a checklist, which was used to provide corrective feedback to SPs.

Residents in the 2 clinics routinely precept patients with attending physicians. We repeatedly informed staff attending physicians about the study design and requested that they be nondirective when advising residents if they suspected a resident was seeing an SP. Residents at each clinic are authorized to order diagnostic tests without an attending physician's cosignature.
We monitored SP detection using an E-mail survey sent 2 to 4 weeks after SP visits; this survey asked residents whether they suspected seeing an SP recently. If residents suspected SPs, we asked them to describe the SP and whether their clinical decisions differed from what they would have done for a real patient.

\section{Measures}

The main study outcome was whether residents ordered requested low-value tests during unannounced SP visits (eg, spinal MRI in the patient with low-back pain). We assessed test ordering by standardized electronic medical record review.

Patient-centered communication was assessed using the Measure of Patient-Centered Communication (MPCC), a validated measure ranging from 0 to 100 (the least to most patient-centered) based on transcribed audio-recordings of the encounters. ${ }^{15}$ Each component has a theoretical range of 0 to 100 , with the total score being an average of the 3 component scores. The MPCC scores physicians on their exploration of the patient's experience of illness (component 1), the psychosocial context (component 2), and physician efforts to find common ground on diagnosis and treatment (component 3). Two trained research assistants coded audiotaped recordings of the visits, resolving disagreements by consensus. Coders were trained by a doctorate-level qualitative researcher over a 3 -month period by discussing and building consensus on blinded coding results for the initial 10 to 15 SP encounters.

Coders also rated the extent to which doctors engaged in the targeted specific physician counseling behaviors: (1) normalization, (2) informing patients about reassuring features of the history and physical examination, (3) explaining that the risks of testing outweighed the benefits, (4) advising watchful waiting, and (5) recommending evidencebased strategies instead of immediate testing. Coding for advising watchful waiting was based on 2 elements: (1) whether the resident recommended that the patient not undergo testing at this time, and (2) whether the resident stated that he or she would reconsider testing at a later point if symptoms or concerns persisted. We specified acceptable evidence-based strategies for each case (eg, dietary calcium intake for women requesting DXA). Each behavior was assessed as present or absent during the encounter. 
We collected data on resident characteristics, including age, sex, postgraduate year, and specialty (family or internal medicine). Residents completed a baseline questionnaire including measures of stress from clinical uncertainty (theoretical score range, 13 to 78 ) and reluctance to disclose such uncertainty (theoretical score range, 9 to 39); higher scores reflected greater stress and reluctance, respectively. ${ }^{21,22}$ These measures capture the extent to which physicians feel anxiety, uneasiness, discomfort, or emotional turmoil when faced with uncertainty in patient care.

\section{Analyses}

Data were analyzed using Stata 14.1 (StataCorp, College Station, TX). Descriptive analyses used $t$ tests or $\chi^{2}$ tests to examine the relationship between test ordering and other study variables. Relationships between communication behaviors and test ordering were assessed using a series of logistic regression analyses within a generalized estimating equation framework, which adjusts standard errors for the nesting of SPs within physicians. A base model (excluding the communication variables) included only the intervention group and case scenario. Subsequent analyses added individual specific communication behaviors or components of the MPCC. Models also included clusters of the specific communication behaviors or the total MPCC score. To facilitate interpretation of the logistic regression parameter estimates, these are reported as adjusted marginal effects (the adjusted prevalence of test ordering associated with each level of the predictor). For each analysis, we report the amount of variance explained in test ordering; explained variance was assessed using McFadden's pseudo- $R^{2} .{ }^{23}$ Our aim was to identify the contribution made by each of the communication measures in explaining the variance in test ordering.

\section{Results}

Of 64 potentially eligible residents, 61 agreed to participate and were randomized, and 59 had at least 1 follow-up visit with an SP. In the 155 encounters with unannounced SPs who requested low-value tests, PCPs ordered spinal MRI for 27\% of the SPs with subacute back pain, DXA for $45 \%$ of the low-risk women requesting DXA screening, and neuroimaging for $9 \%$ of the SPs with uncomplicated headache. Overall, low-value tests were ordered during $26.5 \%$ of unannounced SP visits and during a similar percentage of visits with intervention versus control physicians $(27.3$ vs $25.6 \%$; $P=.82$ ).

Of the communication behaviors, physicians most commonly provided reassurance and recommended evidence-based recommendations, both of which occurred during the most encounters regardless of whether tests were ordered (Table 1). Physicians explained that the risks of tests outweighed the potential benefits in $65 \%$ of visits when no tests were ordered versus $37 \%$ of visits when tests were ordered $(P=.002)$. Physicians advised watchful waiting in $68 \%$ of visits overall but advised watchful waiting more commonly during those encounters for which a test was not ordered (88\% vs $12 \% ; P<.001)$. In bivariate analyses, overall patient-centeredness was not associated with test ordering, nor were individual components of the MPCC. Physician characteristics were not significantly associated with test ordering, including postgraduate year, stress from uncertainty, and reluctance to disclose uncertainty.

In adjusted analyses assessing the contribution of individual and combined communication behaviors to test ordering, physician advice to pursue watchful waiting, an explanation that the risks of testing outweigh the potential benefits, and a discussion of reassuring features of the history and physical examination were each significantly associated with low-value test ordering (Table 2). While the base model (including the study arm and case scenario) explained $9 \%$ of test ordering behavior, the suggestion of watchful waiting by itself produced substantial additional explained variance (pseudo- $R^{2}=$ $53 \%$ ) (Table 2, series 1). Patients advised to pursue watchful waiting were $39 \%$ less likely to have lowvalue tests ordered (adjusted marginal effect, $-38.6 \%$; $95 \%$ confidence interval [CI], $-43.6 \%$ to $-33.6 \%)$. When all communication behaviors except watchful waiting were included, only discussion that the risks of testing outweighed the potential benefits was associated with test ordering (marginal effect, $-22 \%$; 95\% CI, $-32.5 \%$ to $-11.2 \%$ ) (Table 2, series 2), but the total amount of variance explained was much less (pseudo- $R^{2}=$ $16 \%)$. When watchful waiting was included along with other communication behaviors (Table 2, series 3 ), only watchful waiting was significantly associated with test ordering (marginal effect, $-38 \%$; $95 \% \mathrm{CI},-44.3 \%$ to $-31.7 \%$ ), and the inclusion of 
Table 1. Visit and Physician Characteristics and Low-Value Test Ordering During Unannounced Standardized Patient Visits

\begin{tabular}{|c|c|c|c|c|}
\hline \multirow[b]{2}{*}{ Visit and Physician Characteristics } & \multirow[b]{2}{*}{ Total } & \multicolumn{2}{|c|}{ Low-Value Test Ordered } & \multirow[b]{2}{*}{$P$ Valu } \\
\hline & & No & Yes & \\
\hline $\mathrm{N}$ & 155 & 114 & 41 & \\
\hline \multicolumn{5}{|l|}{ Counseling behaviors } \\
\hline Normalization & $10(6.5)$ & $7(6.2)$ & $3(7.3)$ & .80 \\
\hline Risks outweigh benefits & $88(57.1)$ & $73(64.6)$ & $15(36.6)$ & .002 \\
\hline Reassurance & $147(95.5)$ & $110(97.3)$ & $37(90.2)$ & .06 \\
\hline Watchful waiting & $104(67.5)$ & $99(87.6)$ & $5(12.2)$ & $<.001$ \\
\hline Evidence-based strategies & $150(97.4)$ & $111(98.2)$ & $39(95.1)$ & .28 \\
\hline \multicolumn{5}{|l|}{ Patient-centeredness (MPCC), mean (SD) } \\
\hline Component 1 & $40.7(7.8)$ & $40.8(8.0)$ & $40.5(7.4)$ & .82 \\
\hline Component 2 & $47.5(16.7)$ & $47.4(17.6)$ & $47.9(14.0)$ & .86 \\
\hline Component 3 & $43.1(13.4)$ & $42.7(13.6)$ & $44.3(12.8)$ & .52 \\
\hline Total & $43.8(8.2)$ & $43.6(8.5)$ & $44.2(7.4)$ & .69 \\
\hline \multicolumn{5}{|l|}{ Study case } \\
\hline Back pain & $55(35.5)$ & $40(35.1)$ & $15(36.6)$ & $<.001$ \\
\hline DXA & $47(30.3)$ & $26(22.8)$ & $21(51.2)$ & \\
\hline Headache & $53(34.2)$ & $48(42.1)$ & $5(12.2)$ & \\
\hline \multicolumn{5}{|l|}{ Intervention Arm } \\
\hline Intervention & $78(50.3)$ & $58(50.9)$ & $20(48.8)$ & .82 \\
\hline Control & $77(49.7)$ & $56(49.1)$ & $21(51.2)$ & \\
\hline \multicolumn{5}{|l|}{ Physician characteristics } \\
\hline \multicolumn{5}{|l|}{ Postgraduate year } \\
\hline 2 & $85(54.8)$ & $60(52.6)$ & $25(61.0)$ & .62 \\
\hline 3 & $55(35.5)$ & $41(36.0)$ & $14(34.1)$ & \\
\hline 4 & $9(5.8)$ & $8(7.0)$ & $1(2.4)$ & \\
\hline 5 & $6(3.9)$ & $5(4.4)$ & $1(2.4)$ & \\
\hline Stress from uncertainty, mean (SD) & $50.4(10.8)$ & $50.2(10.5)$ & $50.8(11.8)$ & .76 \\
\hline Reluctance to disclose uncertainty, mean (SD) & $25.6(4.7)$ & $25.6(4.7)$ & $25.8(4.3)$ & .79 \\
\hline
\end{tabular}

Data are $\mathrm{n}(\%)$ unless otherwise indicated.

DXA, dual-energy x-ray absorptiometry; MPCC, Measure of Patient-Centered Communication; N, number of encounters (or visits); $\mathrm{SD}$, standard deviation.

all communications behaviors explained little additional variance compared with the model that included watchful waiting alone (pseudo- $R^{2}=56 \%$ vs $53 \%$, respectively).

We similarly assessed the influence of patientcenteredness on low-value test ordering (Table 3). Compared with the variance explained by the base model alone, neither individual components of the MPCC (series 4) nor the overall patient-centeredness of the visit (series 5) explained significant additional variance in test ordering.

Based on surveys conducted within 4 weeks of $\mathrm{SP}$ visits, residents suspected seeing a recent $\mathrm{SP}$ in $59 \%$ of visits (60 of 101 responses to surveys after the visits). In 53 of the 60 visits (88\%) in which residents suspected seeing SPs, they responded that they managed the patient exactly as they would a similar real patient; rates of test ordering were similar for the 7 visits in which residents reported "minor differences" in management compared with visits when they reported managing SPs "exactly alike" real patients ( $14.3 \%$ vs $15.4 \%)$.

\section{Discussion}

In the context of patient requests for low-value diagnostic tests, we found that a physician's suggestion to pursue a watchful waiting strategy was associated with a substantially lower likelihood of test ordering, and that this single communication behavior explained most of the variance in test ordering. After accounting for advice to pursue watchful 
Table 2. Incremental Variance in Low-Value Test Ordering Explained by Specific Physician Communication Behaviors

\begin{tabular}{|c|c|c|c|}
\hline Variables, by Model & $\begin{array}{l}\text { Adjusted Probability of Test Ordering* } \\
\text { (95\% CI) }\end{array}$ & $P$ Value & Pseudo- $R^{2}$ \\
\hline Base model ${ }^{\dagger}$ & - & - & $9.3 \%$ \\
\hline \multicolumn{4}{|l|}{ Series $1^{\ddagger}$} \\
\hline Normalization & $4.7 \%(-23.6$ to $32.9 \%)$ & .75 & $9.3 \%$ \\
\hline Risks outweigh benefits & $-22.6 \%(-33.1$ to 12.0$)$ & $<.001$ & $15.8 \%$ \\
\hline Reassurance & $-19.2 \%(-37.7$ to -0.8$)$ & .04 & $20.3 \%$ \\
\hline Watchful waiting & $-38.6 \%(-43.6$ to -33.6$)$ & $<.001$ & $53.1 \%$ \\
\hline Evidence-based recommendations & $-15.7 \%(-40.3$ to 8.9$)$ & .21 & $9.6 \%$ \\
\hline Series $2^{\S}$ & & & $16.5 \%$ \\
\hline Normalization & $6.4 \%(-23.4$ to $36.2 \%)$ & .67 & \\
\hline Risks outweigh benefits & $-21.9 \%(-32.5$ to $-11.2 \%)$ & $<.001$ & \\
\hline Reassurance & $-14.7 \%(-31.9$ to $2.6 \%)$ & .10 & \\
\hline Evidence-based recommendations & $1.8 \%(-19.9 \%$ to $23.5 \%)$ & .87 & \\
\hline Series $3^{\|}$ & & & $55.7 \%$ \\
\hline Normalization & $9.8 \%(-11.1$ to $30.6 \%)$ & .67 & \\
\hline Risks outweigh benefits & $-6.7 \%(-15.2$ to $1.7 \%)$ & .12 & \\
\hline Reassurance & $5.4(-12.9$ to 23.6$)$ & .57 & \\
\hline Watchful waiting & $-38.0 \%(-44.3$ to $-31.7 \%)$ & $<.001$ & \\
\hline Evidence-based recommendations & $10.3 \%(-2.2$ to $22.7 \%)$ & 0.11 & \\
\hline
\end{tabular}

*Adjusted for the randomized controlled trial intervention.

${ }^{\dagger}$ The base model included study arm and standardized patient (patient with back pain requesting magnetic resonance imaging, woman requesting dual-energy $\mathrm{x}$-ray absorptiometry, patient with headache requesting magnetic resonance imaging). Headache was associated with significantly less test ordering than back pain.

' In series 1, each communication behavior was included individually in separate models with base model variables.

${ }^{\S}$ In series 2, all communication behaviors, except watchful waiting, were included simultaneously together with base model variables.

"In series 3, all communication behaviors were included simultaneously together with the base model.

CI, confidence interval.

waiting, other communication behaviors had no additional impact on test ordering, nor did the overall patient-centeredness of the interaction. Our results suggest that watchful waiting shows promise as a simple counseling strategy that physicians can use to avert low-value test ordering.

While "watchful waiting" has been advocated as a strategy to reduce antibiotic prescribing for acute otitis media and in the management of prostate cancer, ${ }^{17,18}$ we assessed its potential influence in the context of diagnostic testing. In a cluster-randomized trial from the Netherlands, ${ }^{19}$ patients with unexplained symptoms were randomized to immediate blood testing versus 4 weeks of watchful waiting. In general, patients and physicians found watchful waiting acceptable, without adverse effects on patient anxiety. While specific aspects of physician-patient communication may reduce patient anxiety, test ordering does not seem to affect patient satisfaction, and diagnostic testing for patients at low risk of serious disease does little to reassure patients or resolve symptoms. ${ }^{10}$ Our findings suggest that a watchful waiting approach might also be an effective means of reducing low-value diagnostic testing in US primary care settings.

The potential effectiveness of watchful waiting as a counseling strategy is supported by theories of patient autonomy and control. Locus of control theory suggests that patient perception of control may be associated with positive health outcomes. $^{24,25}$ When patients request tests, a negative response from physicians may threaten patients' sense of autonomy or control, whereas a watchful waiting approach may validate patients' concerns and bolster their sense of control over their health and symptoms. ${ }^{26}$ In contrast to watchful waiting, reassurance by itself may be perceived by patients as invalidating their concerns. By boosting patient autonomy, a watchful waiting strategy may also allow physicians to maintain patient satisfaction, ${ }^{25}$ which is major quality improvement target. A watchful waiting approach may also improve the 
Table 3. Incremental Variance in Low-Value Test Ordering Explained by Patient Centered Communication

\begin{tabular}{|c|c|c|c|}
\hline Variables, by Model & $\begin{array}{l}\text { Adjusted Effect on the Probability } \\
\text { of Test Ordering* }(95 \% \mathrm{CI})\end{array}$ & $P$ Value & Pseudo- $R^{2}$ \\
\hline Base model $^{\dagger}$ & - & - & $9.3 \%$ \\
\hline \multicolumn{4}{|l|}{ Series $4^{\ddagger}$} \\
\hline Component 1: patients' experience of illness & $0.1 \%(-1.0 \%, 0.8 \%)$ & .84 & $9.3 \%$ \\
\hline Component 2: psychosocial context & $0.1 \%(-0.3 \%, 0.4 \%)$ & .71 & $9.3 \%$ \\
\hline Component 3: attempt to find common ground & $0.0 \%(-0.5 \%, 0.4 \%)$ & .86 & $9.3 \%$ \\
\hline \multicolumn{4}{|l|}{ Series $5^{\S}$} \\
\hline Total MPCC & $0.0 \%(-0.8 \%, 0.8 \%)$ & .94 & $9.3 \%$ \\
\hline
\end{tabular}

*Adjusted for the randomized controlled trial intervention.

${ }^{\dagger}$ The base model included study arm and standardized patient (patient with back pain requesting magnetic resonance imaging, woman requesting dual-energy $\mathrm{x}$-ray absorptiometry, patient with headache requesting magnetic resonance imaging). Headache was associated with significantly less test ordering than back pain.

${ }^{\ddagger}$ In series 4, each Measure of Patient-Centered Communication (MPCC) component was included individually in separate models with base model variables.

${ }^{\S}$ Series 5 included the total MPCC together with base model variables.

$\mathrm{CI}$, confidence interval.

physician experience by reducing the greater perceived difficulty of visits with patients who request diagnostic tests. ${ }^{8}$

Although previous observational analyses suggested that patient-centered communication may be associated with lower overall diagnostic testing, ${ }^{1,14}$ more patient-centered communication was not associated with test ordering in our study. While prior studies have examined practice patterns among physicians with more versus less patient-centered communication styles, our study examined the association between patientcenteredness and test ordering within a specific visit when a low-value test was requested. It is conceivable that more patient-centered physicians may order fewer diagnostic tests overall, yet within the subset of visits in which low-value tests are requested, physician patient-centeredness may not influence test ordering.

Discretionary care, including test ordering, varies substantially among PCPs. ${ }^{27}$ The national Choosing Wisely ${ }^{\mathrm{TM}}$ initiative seeks to reduce lowvalue testing by disseminating informational materials to providers and the public. ${ }^{3}$ However, the initial impact of the campaign on practice patterns seems to be limited, ${ }^{28}$ and Choosing Wisely is purposefully limited in scope, focusing on short lists of care elements, only some of which involve diagnostic testing. Individually targeted provider education, audit and feedback, and financial incentives have stronger evidence of effectiveness in reducing low-value testing, as do systems-based approaches (eg, computerized clinical decision support). ${ }^{29}$ However, these approaches are again most feasibly applied to short lists of tests and are relatively resource intensive. Teaching physicians how to use watchful waiting may be a generally applicable strategy to reduce the use of a broad range of low-value tests.

This study was limited by several constraints. First, the study had an observational design, and unmeasured confounding is possible. Second, the study included resident physicians at 2 academic practices and may have limited generalizability to other primary care practices or to urgent care or emergency settings. Third, residents often suspected that they were seeing SPs, which may have influenced communication behaviors. However, the similar rate of test ordering among residents who did and did not report differences in SP management imply that SP detection did not substantively confound the observed associations between watchful waiting and lower test ordering. Fourth, attending physicians may have influenced resident communication or testing behavior, although we asked attending physicians to be nondirective when they suspected an SP encounter.

\section{Conclusion}

In this observational study, counseling of patients by resident physicians who used a watchful waiting strategy was associated with less ordering of lowvalue diagnostic tests requested by SPs. This single communication strategy explained a large proportion of the total variance in testing. These findings 
suggest that watchful waiting may be a simple, effective communication strategy for reducing lowvalue testing in primary care.

\section{References}

1. Bertakis KD, Azari R. Patient-centered care is associated with decreased health care utilization. J Am Board Fam Med 2011;24:229-39.

2. Sirovich BE, Woloshin S, Schwartz LM. Too little? Too much? Primary care physicians' views on US health care: a brief report. Arch Intern Med 2011; 171:1582-5.

3. Cassel CK, Guest JA. Choosing wisely: helping physicians and patients make smart decisions about their care. JAMA 2012;307:1801-2.

4. van der Weijden T, van Bokhoven MA, Dinant GJ, van Hasselt CM, Grol RP. Understanding laboratory testing in diagnostic uncertainty: a qualitative study in general practice. Br J Gen Pract 2002;52: 974-80.

5. Tilburt JC, Wynia MK, Sheeler RD, et al. Views of US physicians about controlling health care costs. JAMA 2013;310:380-8.

6. Kravitz RL, Bell RA, Azari R, Kelly-Reif S, Krupat E, Thom DH. Direct observation of requests for clinical services in office practice: what do patients want and do they get it? Arch Intern Med 2003;163: 1673-81.

7. Kravitz RL, Bell RA, Azari R, Krupat E, Kelly-Reif $\mathrm{S}$, Thom D. Request fulfillment in office practice: antecedents and relationship to outcomes. Med Care 2002;40:38-51.

8. Fenton JJ, Franks P, Feldman MD, et al. Impact of patient requests on provider-perceived visit difficulty in primary care. J Gen Intern Med 2015;30: 214-20.

9. van Bokhoven MA, Pleunis-van Empel MC, Koch H, Grol RP, Dinant GJ, van der Weijden T. Why do patients want to have their blood tested? A qualitative study of patient expectations in general practice. BMC Fam Pract 2006;7:75.

10. Rolfe A, Burton C. Reassurance after diagnostic testing with a low pretest probability of serious disease: systematic review and meta-analysis. JAMA Intern Med 2013;173:407-16.

11. Jackson JL, Chamberlin J, Kroenke K. Predictors of patient satisfaction. Soc Sci Med 2001;52:609-20.

12. Stewart M, Ryan RB. The Measure of Patient-Centered Communication (MPCC). London, Ontario, Canada: Thames Valley Family Practice Research Unit and Centre for Studies in Family Medicine; 2001.

13. Paterniti DA, Fancher TL, Cipri CS, Timmermans S, Heritage J, Kravitz RL. Getting to "no": strategies primary care physicians use to deny patient requests. Arch Intern Med 2010;170:381-8.
14. Epstein RM, Franks P, Shields CG, et al. Patientcentered communication and diagnostic testing. Ann Fam Med 2005;3:415-21.

15. Stewart M, Brown JB, Donner A, et al. The impact of patient-centered care on outcomes. J Fam Pract 2000;49:796-804.

16. Deyo RA, Diehl AK, Rosenthal M. Reducing roentgenography use. Can patient expectations be altered? Arch Intern Med 1987;147:141-5.

17. Bill-Axelson A, Holmberg L, Garmo H, et al. Radical prostatectomy or watchful waiting in early prostate cancer. N Engl J Med 2014;370:932-42.

18. McCormick DP, Chonmaitree T, Pittman C, et al. Nonsevere acute otitis media: a clinical trial comparing outcomes of watchful waiting versus immediate antibiotic treatment. Pediatrics 2005;115:1455-65.

19. van Bokhoven MA, Koch $H$, van der Weijden T, et al. Influence of watchful waiting on satisfaction and anxiety among patients seeking care for unexplained complaints. Ann Fam Med 2009;7:112-20.

20. Fenton JJ, Kravitz RL, Jerant A, et al. Promoting patient-centered counseling to reduce use of lowvalue diagnostic tests: a randomized clinical trial. JAMA Intern Med 2016;176:191-7.

21. Gerrity MS, White KP, DeVellis RF, Dittus RS. Physicians' reactions to uncertainty: refining the constructs and scales. Motiv Emot 1995;19:175-91.

22. Gerrity MS, DeVellis RF, Earp JA. Physicians' reactions to uncertainty in patient care. A new measure and new insights. Med Care 1990;28:724-36.

23. McFadden D. Conditional logit analysis of qualitative choice behavior. In: Zarembka P, ed. Frontiers in econometrics. New York, NY: Academic Press; 1974. pp. 120-30.

24. Williams JS, Lynch CP, Voronca D, Egede LE. Health locus of control and cardiovascular risk factors in veterans with type 2 diabetes. Endocrine 2016;51:83-90.

25. Gabay G. Perceived control over health, communication and patient-physician trust. Patient Educ Couns 2015 June 29 [Epub ahead of print].

26. Pilnick A, Dingwall R. On the remarkable persistence of asymmetry in doctor/patient interaction: a critical review. Soc Sci Med 2011;72:1374-82.

27. Sirovich B, Gallagher PM, Wennberg DE, Fisher ES. Discretionary decision making by primary care physicians and the cost of U.S. health care. Health Aff (Millwood) 2008;27:813-23.

28. Rosenberg A, Agiro A, Gottlieb M, et al. Early trends among seven recommendations from the choosing wisely campaign. JAMA Intern Med 2015; 175:1913-20.

29. Stammen LA, Stalmeijer RE, Paternotte E, et al. Training physicians to provide high-value, cost-conscious care: a systematic review. JAMA 2015;314: 2384-400. 